\title{
COMMUTATIVITY OF ONE SIDED S-UNITAL RINGS THROUGH A STREB'S RESULT
}

\author{
MURTAZA A. QUADRI, V.W. JACOB and M. ASHRAF \\ Department of Mathematics \\ Aligarh Muslim University \\ Aligarh - 202002, INDIA
}

(Received October 4, 1994 and in revised form September 19, 1995)

\begin{abstract}
The main theorem proved in the present paper states as follows "Let $m, k, n$ and $s$ be fixed non-negative integers such that $k$ and $n$ are not simultaneously equal to 1 and $R$ be a left (resp right) $s$-unital ring satisfying $\left[\left(x^{m} y^{k}\right)^{n}-x^{s} y, x\right]=0\left(\operatorname{resp}\left[\left(x^{m} y^{k}\right)^{n}-y x^{s}, x\right]=0\right)$ Then $R$ is commutative." Further commutativity of left $s$-unital rings satisfying the condition $x^{t}\left[x^{m}, y\right]-$ $y^{r}[x, f(y)] x^{s}=0$ where $f(t) \in t^{2} Z[t]$ and $m>0, t, r$ and $s$ are fixed non-negative integers, has been investigated Finally, we extend these results to the case when integral exponents in the underlying conditions are no longer fixed, rather they depend on the pair of ring elements $x$ and $y$ for their values These results generalize a number of commutativity theorems established recently
\end{abstract}

KEY WORDS AND PHRASES: Factor subrings, $s$-unital rings, polynomial identities, commutators 1991 AMS SUBJECT CLASSIFICATION CODE: 16 U80.

\section{INTRODUCTION}

Throughout the paper, $R$ will represent an associative ring (may be without unity), $N$ the set of nilpotent elements of $R$, and $U$ the group of units of $R$. For any $x, y$ in $R,[x, y]$ denotes the commutator $x y-y x . R$ is said to be a left (resp. right) s-unital ring if for each $x$ in $R, x \in R x$ (resp. $x \in x R$ ) and $R$ is called $s$-unital in case it is left as well as right $s$-unital. As usual, $Z[t]$ is the totalitý of all polynomials in $t$ over $Z$, the ring of integers. Consider the following ring properties

$\left(\mathrm{P}_{1}\right)$ Suppose that $m, k, n$ and $s$ are non-negative integers with $k$ and $n$ not simultaneously equal to 1 such that $\left[\left(x^{m} y^{k}\right)^{n}-x^{s} y, x\right]=0$ for all $x, y$ in $R$.

$\left(\mathrm{P}_{1}{ }^{*}\right)$ For every $x, y$ in $R$ there exist non-negative integers $m, k, n$ and $s$ with $k$ and $n$ not simultaneously equal to 1 such that $\left[\left(x^{m} y^{k}\right)^{n}-x^{s} y, x\right]=0$

$\left(\mathrm{P}_{2}\right) \quad$ Suppose that $m, k, n$ and $s$ are non-negative integers with $k$ and $n$ not simultaneously equal to 1 such that $\left[\left(x^{m} y^{k}\right)^{n}-y x^{s}, x\right]=0$, for all $x, y$ in $R$

$\left(\mathrm{P}_{2}{ }^{*}\right)$ For every $x, y$ in $R$ there exist non-negative integers $m, k, n$ and $s$ with $k$ and $n$ not simultaneously equal to 1 such that $\left[\left(x^{m} y^{k}\right)^{n}-y x^{s}, x\right]=0$.

$\left(\mathrm{P}_{3}\right)$ For every $y$ in $R$ there exists $f(t)$ in $t^{2} Z[t]$ such that $x^{t}\left[x^{m}, y\right]=y^{r}[x, f(y)] x^{s}$ and $x^{t}\left[x^{n}, y\right]=y^{r}[x, f(y)] x^{s}$ for all $x$ in $R$, where $m \geq 1, n \geq 1, t, r$ and $s$ are fixed non-negative integers with $(m, n)=1$ and at least one of $s$ and $t$ is non-zero 
$\left(\mathrm{P}_{3}{ }^{*}\right)$ For every $x, y$ in $R$ there exist polynomial $f(t)$ in $t^{2} Z[t]$, and non-negative integers $m \geq 1, n \geq 1, t, r, s$ with $(m, n)=1$, and at least one of $s$ and $t$ non-zero such that $x^{t}\left[x^{m}, y\right]=y^{\top}[x, f(y)] x^{s}$ and $x^{t}\left[x^{n}, y\right]=y^{\top}[x, f(y)] x^{s}$

(CH) For every $x, y$ in $R$ there exist $f(t), g(t)$ in $t^{2} Z[t]$ such that $[x-f(x), y-g(y)]=0$

Recently, Wei Zhongxuan [1] proved that if $R$ is a semi prime ring and $m, n$ are fixed positive integers such that either $\left(x^{m} y\right)^{2}-x^{n} y$ or $\left(x^{m} y\right)^{2}-y x^{n}$ is central for all $x, y$ in $R$, then $R$ is commutative The objective of the present paper is to generalize the above result for $s$-unital rings In fact we shall begin with establishing the commutativity of one sided $s$-unital rings satisfying either of the conditions $\left(\mathrm{P}_{1}\right)$ and $\left(\mathrm{P}_{2}\right)$ Further, we shall obtain commutativity of one sided s-unital rings satisfying $\left(\mathrm{P}_{3}\right)$, which extends a result due to Abujabal and Peric [2, Theorem 8]. Finally, commutativity of rings satisfying Chacron's criterion, namely $(\mathrm{CH})$, together with any one of the properties $\left(\mathrm{P}_{1}{ }^{*}\right),\left(\mathrm{P}_{2}{ }^{*}\right)$ and $\left(\mathrm{P}_{3}{ }^{*}\right)$ has been investigated

\section{PRELIMINARY RESULTS}

For the preparation to prove our theorems, let us consider the following types of rings

(a) $)_{1}\left(\begin{array}{cc}G F(p) & G F(p) \\ 0 & 0\end{array}\right), \quad p$ is a prime

(a) $\quad\left(\begin{array}{ll}0 & G F(p) \\ 0 & G F(p)\end{array}\right), \quad p$ is a prime

(b) $M_{\sigma}(K)=\left\{\left(\begin{array}{cc}a & b \\ 0 & \sigma(a)\end{array}\right) / a, b \in K\right.$, where $K$ is a finite field with a non-trivial automorphism $\left.\sigma\right\}$

(c) A non-commutative ring with no nonzero divisors of zero

(d) $S=\langle 1\rangle+T, T$ is a non-commutative subring of $S$ such that $T[T, T]=[T, T] T=0$

In a paper, Streb [3] classified non-commutative rings which has been used effectively as a tool to obtain several commutativity theorems (cf. [4],[5],[6] \& [7]). From the proof of [3, Corollary (1)], it is easy to see that if $R$ is a non-commutative left $s$-unital ring then there exists a factor subring $S$ of $R$ which is of the type (a) $)_{1},(b),(c)$ or (d). This gives us the following lemma that plays a vital role in our subsequent discussion (cf. [8, Meta Theorem]).

LEMMA 1. Let $P$ be a ring property which is inherited by factor subrings If no rings of type (a) , (b), (c) or (d) satisfy $P$, then every left $s$-unital ring satisfying $P$ is commutative

The proofs of the following lemmas can be seen in [9, Theorem], [6, Lemma 1] and [5, Corollary 1] respectively

LEMMA 2. Let $f$ be a polynomial in non-commuting indeterminates $x_{1}, x_{2}, \ldots, x_{n}$ with relatively prime integer coefficients Then the following statements are equivalent:

(i) For any ring $R$ satisfying $f=0$, the commutator ideal of $R$ is a nil ideal

(ii) Every semi prime ring satisfying $f=0$ is commutative.

(iii) For every prime $p$, the ring $(G F(p))_{2}$ of $2 \times 2$ matrices over $G F(p)$ fails to satisfy $f=0$

LEMMA 3. If $R$ is a left $s$-unital ring and not right $s$-unital, then $R$ has a factor subring of type (a)

LEMMA 4. If $R$ is a non-commutative ring satisfying $(\mathrm{CH})$, then there exists a factor subring of $R$ which is of type $(a)_{1}$ or $(b)$

\section{MAIN RESULTS}

We are now well equipped to prove our theorems.

THEOREM 1. If $R$ is a left $s$-unital ring satisfying $\left(\mathrm{P}_{1}\right)$, then $R$ is commutative

PROOF. Let $R$ satisfy $\left(\mathrm{P}_{1}\right)$. As $x=e_{11}+e_{12}$ and $y=e_{12}$ fail to satisfy $\left(\mathrm{P}_{1}\right)$, the ring $R$ is not of type (a) $)_{1}$ So by Lemma 3, $R$ is also right $s$-unital and hence it is $s$-unital Thus in view of Proposition 1 of [10], we may assume that $R$ has the unity 1 
Next, consider the ring $M_{\sigma}(K) \quad$ Let $x=\left(\begin{array}{cc}a & 0 \\ 0 & \sigma(a)\end{array}\right), \quad(a \neq \sigma(a))$ and $y=e_{12} \quad$ Then $\left[\left(x^{m} y^{k}\right)^{n}-x^{s} y, x\right]=-a^{s}(\sigma(a)-a) y \neq 0$, which shows that $R$ is not of type (b)

Now since $x=e_{11}+e_{12}$ and $y=e_{12}$ do not satisfy $\left(\mathrm{P}_{1}\right)$, our Lemma 2 guarantees that the commutator ideal of $R$ is nil and hence no rings of type (c) satisfy $\left(\mathrm{P}_{1}\right)$

Finally, let $S=\langle 1\rangle+T, T$ is non-commutative subring of $S$ such that $T[T, T]=[T, T] T=0$ Choose $a, b \in T$ such that $[a, b] \neq 0$ Then, $[a, b]=(1+a)^{s}[a, b]=\left[1+a,\left((1+a)^{m} b^{k}\right)^{n}\right]=0$, a contradiction. This shows that $R$ is not of type (d)

Thus we have seen that no rings of type $(\mathrm{a})_{1},(\mathrm{~b}),(\mathrm{c})$ or $(\mathrm{d})$ satisfy $\left(\mathrm{P}_{1}\right)$ and so by Lemma $1, R$ is commutative

Since the choice of $x=e_{12}+e_{22}$ and $y=e_{12}$ shows that the rings of type (a) do not satisfy $\left(\mathrm{P}_{2}\right)$, the dual of Lemma 3 forces that every right $s$-unital ring $R$ satisfying $\left(\mathrm{P}_{2}\right)$ is also left $s$-unital and hence $R$ is $s$-unital, and by Proposition 1 of [10], the unity 1 in $R$ may be assumed Now using the same arguments as above, one can easily conclude that $R$ is not of type (a) $,(b),(c)$ or (d), and so the dual of Lemma 1 yields the following.

THEOREM 2. If $R$ is a right $s$-unital ring satisfying $\left(\mathrm{P}_{2}\right)$, then $R$ is commutative.

Our next theorem generalizes a result of Abujabal and Peric [2, Theorem 8]

THEOREM 3. Let $R$ be a left $s$-unital ring satisfying the property $\left(\mathrm{P}_{3}\right)$ Then $R$ is commutative

PROOF. As the property $\left(\mathrm{P}_{3}\right)$ fails to hold for $x=e_{11}+e_{12}$ and $y=e_{12}$, the ring $R$ is not of type (a) 1 Thus, by Lemma $3, R$ is also right $s$-unital and so it is $s$-unital and hence by Proposition 1 of [10] we may assume the unity in $R$

Next consider the ring $M_{\sigma}(K)$, a ring of type (b) Note that $N=K e_{12} \quad$ Thus for $a \in N$ and arbitrary unit $u$, we find that there exists polynomial $f(t)$ in $t^{2} Z[t]$ such that $u^{t}\left[u^{m}, a\right]-a^{r}[u, f(a)] u^{s}=0$ and $u^{t}\left[u^{n}, a\right]-a^{r}[u, f(a)] u^{s}=0$. Since $a^{2}=0$ and $u$ is a unit of $R$, the last two equations imply that $\left[u^{m}, a\right]=0$ and $\left[u^{n}, a\right]=0$, which gives that $[u, a]=0$. Thus for non-central element $a=e_{12}$, we have $\left[u, e_{12}\right]=0$, which forces that $e_{12}$ is central, a contradiction. Hence $R$ cannot be of type (b)

By $\left(P_{3}\right)$, we have

$$
x^{t}\left[x^{m}, y\right]=y^{r}[x, f(y)] x^{s}
$$

On replacing $x$ by $x+1$ in (3.1) and then multiplying it by $x^{s}$, we get

$$
(x+1)^{t}\left[(x+1)^{m}, y\right] x^{s}=y^{r}[x, f(y)](x+1)^{s} x^{s} .
$$

Multiply (3 1) by $(x+1)^{s}$, to get

$$
x^{t}\left[x^{m}, y\right](x+1)^{s}=y^{r}[x, f(y)] x^{s}(x+1)^{s} .
$$

Now, compare (3 2) and (3.3), to get

$$
(x+1)^{t}\left[(x+1)^{m}, y\right] x^{s}=x^{t}\left[x^{m}, y\right](x+1)^{s} .
$$

But (3.4) is a polynomial identity and $x=e_{12}+e_{22}, y=e_{12}$ fail to satisfy the above equality in $(G F(p))_{2}$ Hence by Lemma 2, the commutator ideal of $R$ is nil and so no rings of type (c) satisfy $\left(\mathrm{P}_{3}\right)$

Now let $S=\langle 1\rangle+T$, where $T$ is a non-commutative subring of $S$ such that $T[T, T]=[T, T] T=0$ Let $a, b \in T$ such that $[a, b] \neq 0$ Then there exists $f(t)$ in $t^{2} Z[t]$ such that $m[a, b]=$ $(1+a)^{t}\left[(1+a)^{m}, b\right]=b^{r}[a, f(b)](1+a)^{s}=0$, and $n[a, b]=(1+a)^{t}\left[(1+a)^{n}, b\right]=b^{r}[a, f(b)](1+a)^{s}=0$ This implies that $[a, b]=0$, a contradiction

Thus no rings of type (a) $1,(\mathrm{~b})$, (c) or (d) satisfy $\left(\mathrm{P}_{3}\right)$, and hence, by Lemma $1, R$ is commutative

As in Theorem 1, it can be easily seen that no rings of type $(a)_{1}$ or (b) satisfy $\left(P_{1}{ }^{*}\right)$ Combining this fact with Lemma 4, we obtain the following 
THEOREM 4. Let $R$ be a left $s$-unital ring satisfying the property $\left(\mathrm{P}_{1}{ }^{*}\right)$ Suppose further that $R$ satisfies the property $(\mathrm{CH})$ Then $R$ is commutative

Similarly we can prove the following

THEOREM 5. Let $R$ be a right $s$-unital ring satisfying $\left(\mathrm{P}_{2}^{*}\right)$ Suppose further that $R$ satisfies the property $(\mathrm{CH})$ Then $R$ is commutative

THEOREM 6. Let $R$ be a left $s$-unital ring satisfying the property $(\mathrm{CH})$ Suppose further that $R$ satisfies $\left(\mathrm{P}_{3}{ }^{*}\right)$ Then $R$ is commutative

REMARK. The following example shows that in the hypotheses of Theorems 3 and 6 , the existence of both the conditions in the properties $\left(\mathrm{P}_{3}\right)$ and $\left(\mathrm{P}_{3}{ }^{*}\right)$ is not superfluous (even if $R$ has unity 1 )

EXAMPLE. Let $R=\left\{\left(\begin{array}{lll}a & b & c \\ 0 & a & d \\ 0 & 0 & a\end{array}\right) / a, b, c, d \in G F(2)\right\}$.

Then $R$ is a non-commutative ring with unity satisfying the condition $x^{t}\left[x^{m}, y\right]=y^{r}[x, f(y)] x^{s}$ for a fixed polynomial $f(y)=y^{4}$ and $m=4$, where $t, r, s$ may be any non-negative integers.

We conclude our discussion with the following conjecture

CONJECTURE. Let $R$ be a left $s$-unital ring in which for every $y$ in $R$ there exists a polynomial $f(t)$ in $t^{2} Z[t]$ such that $x^{t}\left[x^{m}, y\right]=y^{r}[x, f(y)] x^{s}$, where $m \geq 1, t, r, s$ are non-negative integers Moreover, if commutators in $R$ are $m$-torsion free, then $R$ is commutative

\section{REFERENCES}

[1] ZHONGXUAN, W., Some commutativity theorems for semiprime rings, Hunan Jiaoyu Xuayuan Xuebao (Ziran Kexue) 7 (1989), 1-4

[2] ABUJABAL, H A S. and PERIC, V., Commutativity of $s$-unital rings through a Streb result, Rad. Mat. 7 (1991), 73-92.

[3] STREB, W., Zur struktur nichtkommutativer ringe, Math. J. Okayama Univ. 31 (1989), 135-140

[4] ASHRAF, M., QUADRI, M.A. and JACOB, V.W., Commutativity of right $s$-unital rings under some polynomial constraints, Tamkang J. Math. 24, No. 1 (1992), 23-28.

[5] KOMATSU, $\mathrm{H}$ and TOMINAGA, H., Chacron's condition and commutativity theorems, Math. J. Okayama Univ. 31 (1989), 101-120.

[6] KOMATSU, H., NISHINAKA, T. and TOMINAGA, H., On commutativity of rings, Rad. Mat. 6 (1990), 303-311.

[7] NISHINAKA, T., A commutativity theorem for rings, Rad. Mat. 6 (1990), 357-359

[8] KOMATSU, H and TOMINAGA, H., Some commutativity theorems for left $s$-unital rings, Results in Math. 15 (1989), 335-342

[9] KEZLAN, T.P , A note on commutativity of semiprime PI-rings, Math. Japonica 27 (1982), $267-$ 268

[10] HIRANO, Y., KOBAYASHI, Y. and TOMINAGA, H., Some polynomial identities and commutativity of $s$-unital rings, Math. J. Okayama Univ. 24 (1982), 7-13 


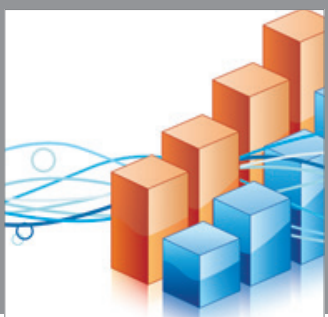

Advances in

Operations Research

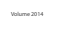

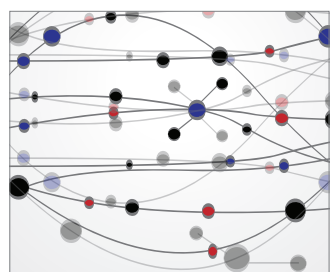

\section{The Scientific} World Journal
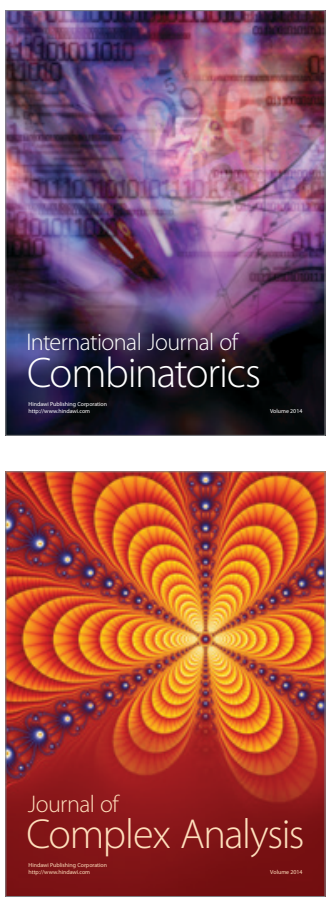

International Journal of

Mathematics and

Mathematical

Sciences
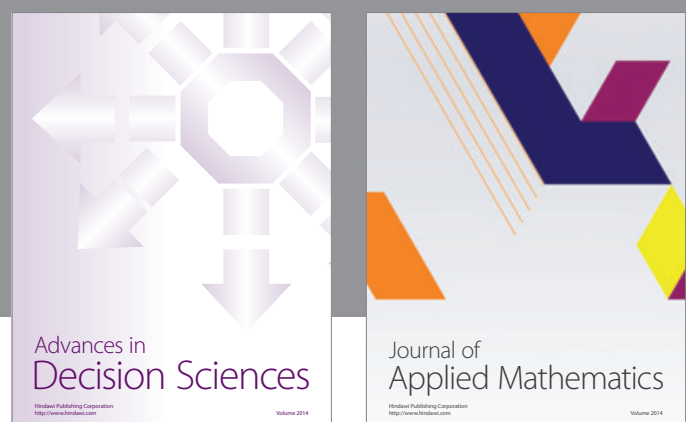

Journal of

Applied Mathematics
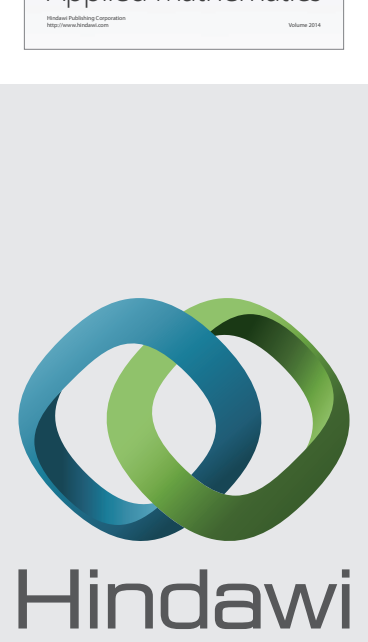

Submit your manuscripts at http://www.hindawi.com
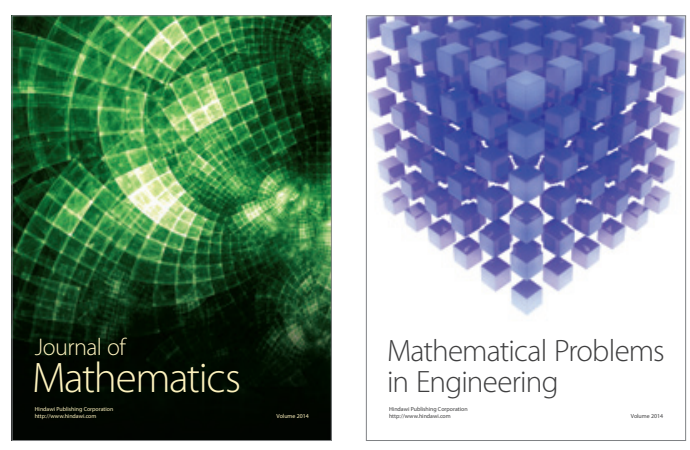

Mathematical Problems in Engineering
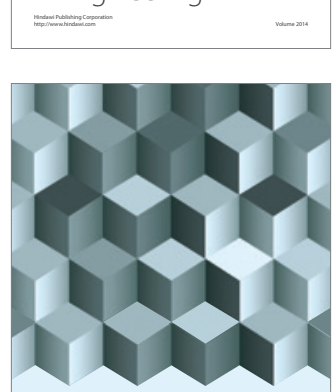

Journal of

Function Spaces
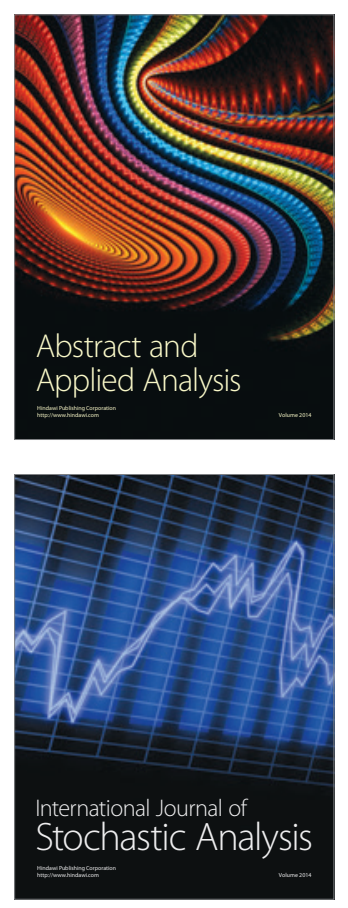

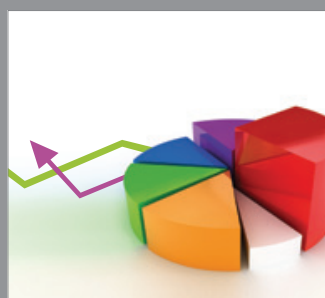

ournal of

Probability and Statistics

Promensencen
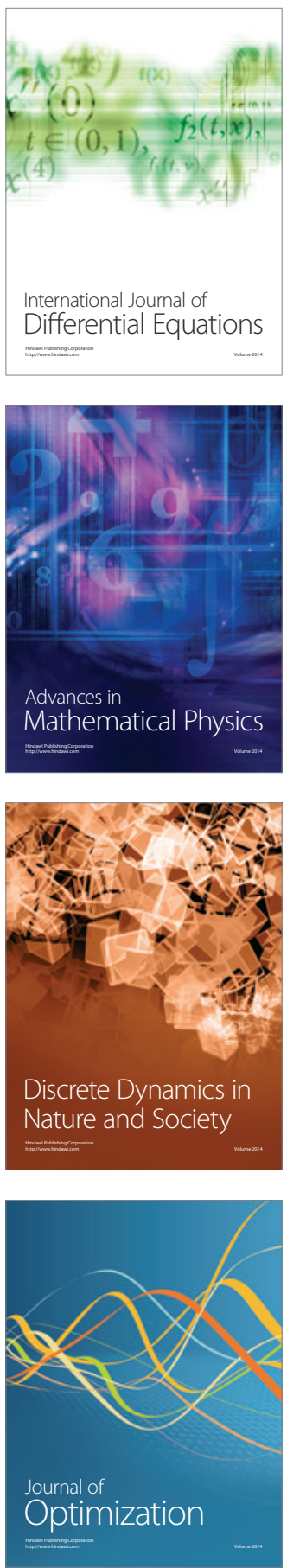